\title{
Integrated analysis of autophagy-related genes(ATGs) reveals the prognostic value of oral squamous cell carcinoma
}

Guangzhao Huang ( $1294329270 @ q q . c o m$ )

Southern Medical University Nanfang Hospital

\section{Zhi-yun Li}

Southern Medical University Nanfang Hospital

\section{Yu Rao}

Southern Medical University Nanfang Hospital

Xiao-zhi Lv

Southern Medical University Nanfang Hospital

Research article

Keywords: Autophagy, ATG12, BID, prognosis,bioinformatics, OSCC

Posted Date: March 13th, 2020

DOI: https://doi.org/10.21203/rs.3.rs-17099/v1

License: (c) (i) This work is licensed under a Creative Commons Attribution 4.0 International License. Read Full License 


\section{Abstract}

Background: Increasing evidence demonstrated that autophagy paly a crucial role in initiation and progression of OSCC. The aim of this study was to explore the prognostic value of autophagy-related genes(ATGs) in patients with OSCC. RNA-seq and clinical data were downloaded from TCGA database following extrating ATGs expression profiles. Then, differentially expressed analysis was performed in R software EdgeR package, and the potential biological function of differentially expressed ATGs were explored by GO and KEGG enrichment analysis. Furthermore, a risk score model based on ATGs was constructed to predict the overall survival. Moreover, univariate, multivariate cox regression and survival analysis were used to select autophagy related biomarkers which were identified by RT-qPCR in OSCC cell lines, OSCC tissues and matched normal mucosal tissues.

Results: Total of 232 ATGs were extrated and 37 genes were differentially expressed in OSCC. GO and KEGG analysis indicated that these differentially expressed genes were mainly located in autophagosome membrane, and associated with apoptosis, platinum drug resistance, ErbB signaling pathway and TNF signaling pathway. Furthermore, a risk score model including 9 variables was constructed and subsequently identified with univariate, multivariate cox regression, survival analysis and Receiver Operating Characteristic curve(ROC). Moreover, ATG12 and BID were identified as potential autophagy related biomakers.

Conclusion: This study successfully constructed a risk model to predict the prognosis of patients with OSCC, and the risk score may be as a independent prognostic biomarker in OSCC. ATG12 and BID were identified as potential biomarkers in tumor diagnosis and treatment of OSCC.

\section{Introduction}

Oral squamous cell carcinoma (OSCC) is the most common head and neck squamous cell carcinoma and one of the most common problems in many parts of the world[1]. Smoking, drinking, betel nut and human papillomavirus(HPV) infection are most conmon risk factors[2]. Despite advances in medical equipment and treatment, the overall survival rate of OSCC is still unsatisfied. It mainly owed to the lack of effective biomarkers for early-stage diagosis, recurrence and lymph node metastases[3]. Therefore, it is crucial and pressing to identify the effective biomarkers and therapeutic targets for OSCC to improve the overall survival.

Autophagosome is formed by encapsulating part of the cytoplasm and components such as organelles and proteins. Then, autophagosome is fused with lysosomes to form autophagosome, which degrades the wrapped contents, so as to realize the metabolic needs of the cell itself and the renewal of some organelles. In summary, autophagy is a lysosomal-mediated catabolic complex process which involves the cytoplasmic organelles and proteins to maintain metabolism and homeostasis in cells[4]. Recently, increasing number of evidence showed that autophagy dysregulation played a crucial role in a variety of human malignant tumors, including colorectal cancer[5], renal cell carcinoma[6], non-small cell lung 
cancer[7] and so on. Numerous evidence showed that autophagy may play a significant role in OSCC carcinogenesis. Such as autophagy mediated cell apoptosis to promote tumor progression via the AKT/mTOR pathway in OSCC [8]. In addition, dysregulation of autophagy was relevant to tumorigenesis and prognosis in OSCC[9]. However, the role of whole subsets of ATGs in OSCC has not been explored. Therefore, comprehensive analysis of ATGs may reveal the prognostic value and potential therapeutic targets for OSCC diagnosis and treatment.

The purpose of this study was to analyze the differential expression of ATGs in OSCC and establish a cox regression model to predict the survival of OSCC patients. Furthermore, survival analysis was performed to identify this cox regression model. The present study provides novel insight for better understanding of ATGs in OSCC and useful resource for identifcation of novel biomarkers of OSCC.

\section{Results}

\subsection{Identification of differentially expressed ATGs}

316 OSCC patients and 32 normal controls RNA-seq and corresponding clinical data were downloaded from TCGA database. Then, 232 autophagy related genes expression level were extracted from the transcriptome data. Subsequently, differentially expressed analysis was performed in R software EdgeR package(Figure1A,B,C).Finally, 11 upregulated and 26 downregulated autophagy related genes were sorted out With the cut-off criteria |log2FoldChange| $>1$ and FDR $<0.01$.

\subsection{Functional Analysis of differentially expressed autophagy related genes}

GO enrichment analysis indicated that these genes were mainly located in autophagosome membrane, and associated with cytokine receptor binding and regulation of apoptotic signaling pathway(Figure2A,C). In addition, KEGG pathway analyses showed that most of significant autophagy related genes were enriched in apoptosis, platinum drug resistance, ErbB signaling pathway and TNF signaling pathway(Figure2B,D).

\subsection{Establishment of cox regression model}

Through univariate and multivariate cox regression analysis, total of 9 variables including BID, ATG12, BAK1, SPHK1, NKX2-3, ATIC, LAMP1, ATF6, BNIP3 were enrolled in cox model(Figure3A,B). And rish_score $=\left(0.35502{ }^{\star} \mathrm{BID}\right)+\left(0.69633^{\star} \mathrm{ATG} 12\right)+(0.22561 * \mathrm{BAK} 1)-(0.24922 * \mathrm{SPHK} 1)-(0.66016 * \mathrm{NKX} 2-3)+$ $(0.30945 * A T I C)+(0.30416 *$ LAMP1 $)+(0.50726 * A T F 6)+(0.26573 * B N I P 3)$. Subsequently, OSCC patients in TCGA database were divided into high risk group and low risk group according to cox formula median.Survival analysis indicated that overall survival of high risk core group patients were significantly lower than the low-risk group(Figure3C). Moreover, the expression level of protective ATGs in the low risk group was higher than that in the high risk group.On the contrary, risky genes were lower in high risk group (Figure3D). The risk score combined with survival data were visualized in $\mathrm{R}$ software (Figure3E,F).

\subsection{Identification of cox regression model}


Firstly, the ROC was plotted, and its area under the curve(AUC) is 0.75 which markedly higher than other clinical characteristics(Figure4A). Furthermore, risk score in early stage was significantly lower than terminal stage(Figure4B) indicating that risk score on basis of ATGs may realize early diagnosis in OSCC. Moreover, univariate and multivariate cox regression analysis indicating that risk score may be regarded as an independent prognostic factor(Figure4C,D). Furthmore, the survival curve of ATGs relevant to OSCC overall survival were ploted in R software. And ATG12 and BID were selected as 2 potential independent biomarkers of OSCC in the cox regression model according to univariate(Figure3A), multivariate cox regression(Figure3B) and survival analysis (Figure4E,F) .

\subsection{Survival analysis}

The prognostic values of the risk score for different clinicopathological parameters including age, gender, $T$ and $\mathrm{N}$ in TNM system, grade and stage were further investigated. $\mathrm{M}$ classification in TNM system were excluded because of numerous data missing. Survival analysis demonstrated that low risk group had significantly longer overall survival than high risk group in the stratification analysis based on age, gender, $\mathrm{T}, \mathrm{N}$, grade and stage(Figure5).

\subsection{Identification of potential independent prognostic biomakers}

Comprehensive bioinformatics indicated that ATG12 and BID were associated with overall survival in OSCC. And univariate and multivariate cox regression showed that ATG12 and BID might be selected as potential independent prognostic biomarkers in our study. Therefore, ATG12 and BID genes expression were validated in OSCC cell lines and tissues by qRT-PCR. Our results revealed that ATG12 and BID were upregulation in OSCC cell lines and tissues than MNTs, which were similar with the results in TCGA database (Figure6A,B). Unfortunately, the correlation between ATG12,BID and clinical parameters showed no significant difference (Table1, Table2).

\section{Discussion}

Owing to low 5-year overall survival rates and high recurrence rate [10], it is crucial to explore the effective therapeutic targets to improve the overall survival in OSCC. Recently, increasing number of studies indicated that autophagy may play a significant role in the genesis and development of OSCC [11-13]. However, the role of whole subsets of ATGs in OSCC keep unclear. Therefore, our study aimed to analyze the ATGs comprehensively to reveal the potential therapeutic targets of OSCC.

Total of 232 ATGs were enrolled in this study and 37 genes were differentially expressed in OSCC. According to $\mathrm{GO}$ enrichment analysis, differentially expressed ATGs were accociated with apoptosis which was relevant to cancer progression[14, 15]. In addition, KEGG enrichment analysis showed that these ATGs were relevant to platinum drug resistance, ErbB signaling pathway and TNF signaling pathway. Among these pathways, platinum drug resistance may play a important role in OSCC treatment strategies[16]. In addition, ErbB signaling pathway was also related to head and neck squamous cell carcinoma (HNSCC) treatment[17]. 
In the present study, we established a risk score model through bioinformatics analysis

of 37 differentially expressed ATGs to predict overall survival of OSCC. Finally, 9 ATGs were selected in regression model and it can accurately predict overall survival. Meanwhile, univariate, multivariate cox regression and survival analysis based on stratification analysis indicated that risk score can be regarded as independent prognostic factor. And it also can distinguish early stage tumors and terminal stage tumors. Therefore, the risk score might hold potential in OSCC early diagnosis, forecast and therapy. ATG12 is the human homolog of a yeast protein involved in autophagy. Mizushima $\mathrm{N}$ et al demostated that ATG12 system is well conserved and may function in autophagy also in human cells[18]. Upregulation of ATG12 was correlated with advanced TNM stage in gastric cancer[19] but downregulation of ATG12 induced oncosis in tumor cells[20]. BID was found on chromosome 22q11.21 and encodes for a protein associated with apoptosis, which is heterodimerized with apoptotic activator $\mathrm{BAX}$ or negative apoptotic regulator BCL2. associated with poor prognosis of clear cell Renal Cell Carcinoma (ccRCC) patients in a autophagy related manner[21]. Unfortunately, few studies have reported the relationship between ATG12,BID and OSCC. In our study, bioinformatics analysis indicated that ATG12 and BID were potential independent prognostic biomarkers and subsequentially validated in OSCC cell lines and specimans. Therefore, ATG12 and BID would play a role as novel biomarkers in tumor diagnosis and treatment of OSCC. However, the correlation between ATG12,BID and clinical parameters showed no significant difference. The exact role of ATG12 and BID in OSCC need further study. In addtion, BAK1, SHPK1, LAMP1, ATIC were also associated with viarious cancers in a autophagydependent manner [22][23][24][25]. Ney PA demonstrated that BNIP3 was accociated with mitochondrial autophagy[26]. However, there are fewer studies indicated that other genes in the risk formula play important roles in the development and progression of cancer in a autophagy manner. Numerous researches reported that autophagy played a important role in OSCC [11, 27]. And these genes regulated autophagy in a variety of ways, whether these autophagy related genes were also related to OSCC development and progression in a autophagy manner remains to be further investigated.

We ananlyzed autophagy related genes comprehensively indicating that these differentially expressed genes were relevant to OSCC initiation and progression. Furthermore, a 9 autophagy related gene risk score model was successfully constructed which is positively associated with overall survival in OSCC. And this risk formula might provided potential in OSCC early diagnosis, forecast and therapy. However, there are sevearl limitations in our study. First, the sample numbers in TCGA database are markedly inadequate. Furthermore, it is necessary for further validation in our following research.

\section{Conclusion}

This study successfully constructed a risk model to predict the prognosis of patients with OSCC through conprehensively analyzing ATGs, and the risk score may be as a independent prognostic biomarker in OSCC. Furthermore, ATG12 and BID were identified as potential biomarkers in tumor diagnosis and treatment of OSCC. 


\section{Materials And Methods}

\subsection{Data downloading}

All transcriptome profiling of OSCC was downloaded from The Cancer Genome Atlas(TCGA) database(https://portal.gdc.cancer.gov/). Owing to its half-baked clinical data, 3 samples were excluded. Finally, 316 OSCC samples and 32 normal controls were enrolled in our study. Subsequently, total of 232 autophagy related genes were extracted from transcriptome profile in $\mathrm{R}$ software(Version 3.6.1). Subsequently, differentially expressed analysis was performed in R software EdgeR package with the cutoff criteral |log2 (fold change [FC])|>2.0 and FDR(adjusted P-value) $<0.01$.

\section{$5.2 \mathrm{GO}$ and KEGG analysis}

Gene Ontology (GO) was perfomed to analyze these differentially expressed ATGs in DAVID (https://david.ncifcrf.gov/). In addition, the Kyoto Encyclopedia of Genes and Genomes (KEGG) pathway enrichment analysis was employed to annotate the functions. The significance level of $p<0.05$ was taken as the cut-off standard.

\subsection{Construction of cox regression model}

The differentially expressed ATGs were transformed and normalized in a log2( $x+1)$ manner[28]. Firstly, univariate cox regression was performed to screen prognosis relevant genes with the cut-off criteria pvalue $<0.05$ ). Then, stepwise regression was used to construct the cox risk model according to these prognostic genes to predict the overall survival of OSCC patients. Furthermore, the risk score for each OSCC patient was visualized in $\mathrm{R}$ software on basis of regression model.

\subsection{Identification of cox regression model}

The predictive power of the signature was evaluated using the Receiver operating characteristic(ROC). In addition, univariate and multivariate cox regression were used to analyze the clinical characteristics including age, gender, grade, stage, TNM and risk score. Distant metastasis was excluded owing to numerous missing datum.

\subsection{Survival analysis based on cox model}

According to cox model, OSCC patients in TCGA were divided into low and high risk group. Kaplan-Meier survival curves and stratification analysis along with a logrank $\mathrm{p}$ test were applied to validate its accuracy in R software survival package.

\subsection{Collection of OSCC specimens}

50 cases of OSCC tissue and matched normal mucosal tissues (MNTs) were collected from Nanfang Hospital, Southern Medical University. MNT at least $1.5 \mathrm{~cm}$ from the edge of the tumor was defined as a 
normal control. All 50 patients with OSCC were confirmed by pathology. All patients agreed to the study and signed the consent form.

\subsection{Cell culture}

OSCC cell lines(SCC9, SCC 15, SCC 25,Cal27) and HOK were obtained from Institute of Antibody Engineering, Southern Medical University (Guangzhou, China). Cell lines SCC15 and SCC25 were seeded in DMEM, SCC9 in DMEM/F12 and Cal 27 in a-MEM containing 10\% fetal bovine serum(FBS) and incubated at $37{ }^{\circ} \mathrm{C}$ with $5 \% \mathrm{CO}_{2}$.

\subsection{RNA extraction and quantitative real-time PCR (qRT-PCR)}

Total RNAs of tissues and OSCC cells were extracted with TRIzol reagent and reversed to cDNA. Crucial ATGs expresion level were deteted by qRT-PCR. The sequences of the PCR primers used were as follows: ATG12, forward 5'-CTGCTGGCGACACCAAGA AA- 3'and reverse 5'-CGTGTTCGCTCTACTGCCC-3'; BID, forward 5'-ATGGACCGTAGCATCC CTCC-3' and reverse 5'-GTAGGTGCGTAGGTTCTGGT- 3'; GAPDH, forward 5'-CGCTGA GTACGTCGTGGAGTC-3' and reverse 5'-GCTGATGATCTT GAGGCTGTTGTC-3'.

\subsection{Statistical Analysis}

SPSS23.0 software (IBM) was used for statistical analysis. P value less than 0.05 was considered statistically significant.

\section{Abbreviations}

OSCC,oral squamous cell carcinoma; ATGs, autophagy-related genes; ROC, Receiver Operating Characteristic curve; GO, Gene Ontology; KEGG, Kyoto Encyclopedia of Genes and Genomes; TCGA, The Cancer Genome Atlas.

\section{Declarations}

Publication Ethics: This study was approved by NanFang Hospital ethics committee (AF/SC-09/03.2).

Declaration of interest: There are no conflicts of interests.

Consent for publication: All of authors were consent for publication.

Data Availability Statement: All data for this study are available from corresponding authors if required.

Funding: This research was supported by the National Natural Science Foundation of China (81472536); the Science and Technology Planning Project of Guangdong Province (2017A020215181;2014A020212440); Project of Educational Commission of Guangdong Province of China (2018KTSCX026); the Scientific Research Planning Project of Southern Medical University (CX2018N016) and the Presidential Foundation of the Nanfang Hospital 
Authors' contributions: Xiaozhi Lv designed the study. Guangzhao Huang performed bioinformatics and experiments. Zhi-yun Li performed bioinformatics and expreiments. Yu Rao analyzed the data and collected OSCC specimens. Xiaozhi Lv obtained the funding. Guangzhao Huang and Zhi-yun Li prepared the figures. Guangzhao Huang wrote the manuscript. Xiaozhi Lv supervised the study. All authors read and approved the final manuscript.

Acknowledgements: Not applicable.

\section{References}

1. Morris L, Chandramohan R, West L, Zehir A, Chakravarty D, Pfister DG, Wong RJ, Lee NY, Sherman EJ, Baxi SS et al: The Molecular Landscape of Recurrent and Metastatic Head and Neck Cancers: Insights From a Precision Oncology Sequencing Platform. JAMA ONCOL 2017, 3(2):244-255.

2. Ang KK, Harris J, Wheeler R, Weber R, Rosenthal DI, Nguyen-Tan PF, Westra WH, Chung CH, Jordan $\mathrm{RC}$, Lu C et al: Human papillomavirus and survival of patients with oropharyngeal cancer. $N$ Engl $J$ Med 2010, 363(1):24-35.

3. Chen YJ, Chang JT, Liao CT, Wang HM, Yen TC, Chiu CC, Lu YC, Li HF, Cheng AJ: Head and neck cancer in the betel quid chewing area: recent advances in molecular carcinogenesis. CANCER SCI 2008, 99(8):1507-1514.

4. Klionsky DJ, Abdalla FC, Abeliovich H, Abraham RT, Acevedo-Arozena A, Adeli K, Agholme L, Agnello $\mathrm{M}$, Agostinis P, Aguirre-Ghiso JA et al: Guidelines for the use and interpretation of assays for monitoring autophagy. AUTOPHAGY 2012, 8(4):445-544.

5. Liu S, Lin H, Wang D, Li Q, Luo H, Li G, Chen X, Li Y, Chen P, Zhai B et al: PCDH17 increases the sensitivity of colorectal cancer to 5 -fluorouracil treatment by inducing apoptosis and autophagic cell death. Signal Transduct Target Ther 2019, 4:53.

6. Chow PM, Liu SH, Chang YW, Kuo KL, Lin WC, Huang KH: The covalent CDK7 inhibitor THZ1 enhances temsirolimus-induced cytotoxicity via autophagy suppression in human renal cell carcinoma. CANCER LETT 2019.

7. Liu Y, Wu L, Ao H, Zhao M, Leng X, Liu M, Ma J, Zhu J: Prognostic implications of autophagyassociated gene signatures in non-small cell lung cancer. Aging (Albany NY) 2019, 11.

8. Yang $Y$, Chen D, Liu H, Yang K: Increased expression of IncRNA CASC9 promotes tumor progression by suppressing autophagy-mediated cell apoptosis via the AKT/mTOR pathway in oral squamous cell carcinoma. CELL DEATH DIS 2019, 10(2):41. 
9. Liu PF, Chang HW, Cheng JS, Lee HP, Yen CY, Tsai WL, Cheng JT, Li YJ, Huang WC, Lee CH et al: Map1lc3b and Sqstm1 Modulated Autophagy for Tumorigenesis and Prognosis in Certain Subsites of Oral Squamous Cell Carcinoma. J CLIN MED 2018, 7(12).

10. Zini A, Czerninski R, Sgan-Cohen HD: Oral cancer over four decades: epidemiology, trends, histology, and survival by anatomical sites. J ORAL PATHOL MED 2010, 39(4):299-305.

11. Khan T, Relitti N, Brindisi M, Magnano S, Zisterer D, Gemma S, Butini S, Campiani G: Autophagy modulators for the treatment of oral and esophageal squamous cell carcinomas. MED RES REV 2019.

12. Liu PF, Chang HW, Cheng JS, Lee HP, Yen CY, Tsai WL, Cheng JT, Li YJ, Huang WC, Lee CH et al: Map1lc3b and Sqstm1 Modulated Autophagy for Tumorigenesis and Prognosis in Certain Subsites of Oral Squamous Cell Carcinoma. J CLIN MED 2018, 7(12).

13. Gao L, Dou ZC, Ren WH, Li SM, Liang X, Zhi KQ: CircCDR1as upregulates autophagy under hypoxia to promote tumor cell survival via AKT/ERK(1/2)/mTOR signaling pathways in oral squamous cell carcinomas. CELL DEATH DIS 2019, 10(10):745.

14. Park BS, Choi NE, Lee JH, Kang HM, Yu SB, Kim HJ, Kang HK, Kim IR: Crosstalk between Fisetininduced Apoptosis and Autophagy in Human Oral Squamous Cell Carcinoma. J CANCER 2019, 10(1):138-146.

15. He ZJ, Zhu FY, Li SS, Zhong L, Tan HY, Wang K: Inhibiting ROS-NF-kappaB-dependent autophagy enhanced brazilin-induced apoptosis in head and neck squamous cell carcinoma. FOOD CHEM TOXICOL 2017, 101:55-66.

16. Hung CC, Chien CY, Chu PY, Wu YJ, Lin CS, Huang CJ, Chan LP, Wang YY, Yuan SF, Hour TC et al: Differential resistance to platinum-based drugs and 5-fluorouracil in p22phox-overexpressing oral squamous cell carcinoma: Implications of alternative treatment strategies. Head Neck 2017, 39(8):1621-1630.

17. Cohen RB: Current challenges and clinical investigations of epidermal growth factor receptor (EGFR)and ErbB family-targeted agents in the treatment of head and neck squamous cell carcinoma (HNSCC). CANCER TREAT REV 2014, 40(4):567-577.

18. Mizushima N, Sugita $H$, Yoshimori T, Ohsumi Y: A new protein conjugation system in human. The counterpart of the yeast Apg12p conjugation system essential for autophagy. J BIOL CHEM 1998, 273(51):33889-33892.

19. Cao QH, Liu F, Yang ZL, Fu XH, Yang ZH, Liu Q, Wang L, Wan XB, Fan XJ: Prognostic value of autophagy related proteins ULK1, Beclin 1, ATG3, ATG5, ATG7, ATG9, ATG10, ATG12, LC3B and p62/SQSTM1 in gastric cancer. AM J TRANSL RES 2016, 8(9):3831-3847.

20. Liu H, He Z, Germic N, Ademi H, Frangez Z, Felser A, Peng S, Riether C, Djonov V, Nuoffer JM et al: ATG12 deficiency leads to tumor cell oncosis owing to diminished mitochondrial biogenesis and reduced cellular bioenergetics. CELL DEATH DIFFER 2019.

21. Wan B, Liu B, Yu G, Huang Y, Lv C: Differentially expressed autophagy-related genes are potential prognostic and diagnostic biomarkers in clear-cell renal cell carcinoma. Aging (Albany NY) 2019, 
11(20):9025-9042.

22. Pedro JM, Wei Y, Sica V, Maiuri MC, Zou Z, Kroemer G, Levine B: BAX and BAK1 are dispensable for ABT-737-induced dissociation of the BCL2-BECN1 complex and autophagy. AUTOPHAGY 2015, 11(3):452-459.

23. Liu H, Ma Y, He HW, Zhao WL, Shao RG: SPHK1 (sphingosine kinase 1) induces epithelialmesenchymal transition by promoting the autophagy-linked lysosomal degradation of CDH1/Ecadherin in hepatoma cells. AUTOPHAGY 2017, 13(5):900-913.

24. Chen H, Li L, Hu J, Zhao Z, Ji L, Cheng C, Zhang G, Zhang T, Li Y, Chen H et al: UBL4A inhibits autophagy-mediated proliferation and metastasis of pancreatic ductal adenocarcinoma via targeting LAMP1. J Exp Clin Cancer Res 2019, 38(1):297.

25. Li M, Jin C, Xu M, Zhou L, Li D, Yin Y: Bifunctional enzyme ATIC promotes propagation of hepatocellular carcinoma by regulating AMPK-mTOR-S6 K1 signaling. CELL COMMUN SIGNAL 2017, 15(1):52.

26. Ney PA: Mitochondrial autophagy: Origins, significance, and role of BNIP3 and NIX. Biochim Biophys Acta 2015, 1853(10 Pt B):2775-2783.

27. Gao L, Dou ZC, Ren WH, Li SM, Liang X, Zhi KQ: CircCDR1as upregulates autophagy under hypoxia to promote tumor cell survival via AKT/ERK(1/2)/mTOR signaling pathways in oral squamous cell carcinomas. CELL DEATH DIS 2019, 10(10):745.

28. Huang GZ, Wu QQ, Zheng ZN, Shao TR, Lv XZ: Identification of Candidate Biomarkers and Analysis of Prognostic Values in Oral Squamous Cell Carcinoma. FRONT ONCOL 2019, 9:1054.

\section{Tables}


Table1. Correlation between ATG12 expression and clinical parameters in OSCC patients $(n=50)$

\begin{tabular}{|c|c|c|c|c|}
\hline \multirow[t]{3}{*}{ Parameters } & \multicolumn{3}{|c|}{ ATG12(\%) } & \multirow{3}{*}{$\mathrm{p}$ value } \\
\hline & $\mathrm{n}$ & High & Low & \\
\hline & \multicolumn{3}{|c|}{ expression expression } & \\
\hline Age(years) & & & & 0.5607 \\
\hline$>=60$ & 31 & 14 & 17 & \\
\hline$<60$ & 19 & 11 & 8 & \\
\hline Gender & & & & 0.3926 \\
\hline male & 28 & 17 & 11 & \\
\hline female & 22 & 10 & 12 & \\
\hline Stage & & & & 0.5514 \\
\hline$\square+\square$ & 32 & 20 & 12 & \\
\hline$\square+\square$ & 18 & 9 & 9 & \\
\hline $\mathrm{T}$ classification & & & & 0.3580 \\
\hline $\mathrm{T} 1+\mathrm{T} 2$ & 35 & 15 & 20 & \\
\hline $\mathrm{T} 3+\mathrm{T} 4$ & 15 & 9 & 6 & \\
\hline $\mathrm{N}$ classification & & & & 0.1906 \\
\hline $\mathrm{N} 0+\mathrm{N} 1$ & 37 & 19 & 18 & \\
\hline $\mathrm{N} 2+\mathrm{N} 3$ & 13 & 10 & 3 & \\
\hline Distant metastasi & & & & 0.7067 \\
\hline M0 & 40 & 28 & 12 & \\
\hline M1 & 10 & 6 & 4 & \\
\hline
\end{tabular}

Table2. Correlation between BID expression and clinical parameters in OSCC patients $(n=50)$

\begin{tabular}{|c|c|c|c|c|}
\hline \multirow[t]{2}{*}{ Parameters } & \multicolumn{3}{|c|}{$\mathrm{BID}(\%)$} & \multirow[b]{2}{*}{$\mathrm{p}$ value } \\
\hline & $\mathrm{n}$ & $\begin{array}{l}\text { High } \\
\text { pressic }\end{array}$ & $\begin{array}{l}\text { Low } \\
\text { pression }\end{array}$ & \\
\hline Age(years) & & & & 0.5675 \\
\hline$>=60$ & 29 & 12 & 17 & \\
\hline$<60$ & 21 & 11 & 10 & \\
\hline Gender & & & & 0.5647 \\
\hline male & 32 & 19 & 13 & \\
\hline female & 18 & 9 & 9 & \\
\hline Stage & & & & 0.3659 \\
\hline$\square+\square$ & 33 & 21 & 12 & \\
\hline$\square+\square$ & 17 & 8 & 9 & \\
\hline $\mathrm{T}$ classification & & & & 0.2410 \\
\hline $\mathrm{T} 1+\mathrm{T} 2$ & 27 & 15 & 12 & \\
\hline $\mathrm{T} 3+\mathrm{T} 4$ & 23 & 17 & 6 & \\
\hline $\mathrm{N}$ classification & & & & 0.7742 \\
\hline $\mathrm{N} 0+\mathrm{N} 1$ & 31 & 18 & 13 & \\
\hline $\mathrm{N} 2+\mathrm{N} 3$ & 19 & 10 & 9 & \\
\hline Distant metastasi & & & & 0.7517 \\
\hline M0 & 36 & 20 & 16 & \\
\hline M1 & 14 & 9 & 5 & \\
\hline
\end{tabular}




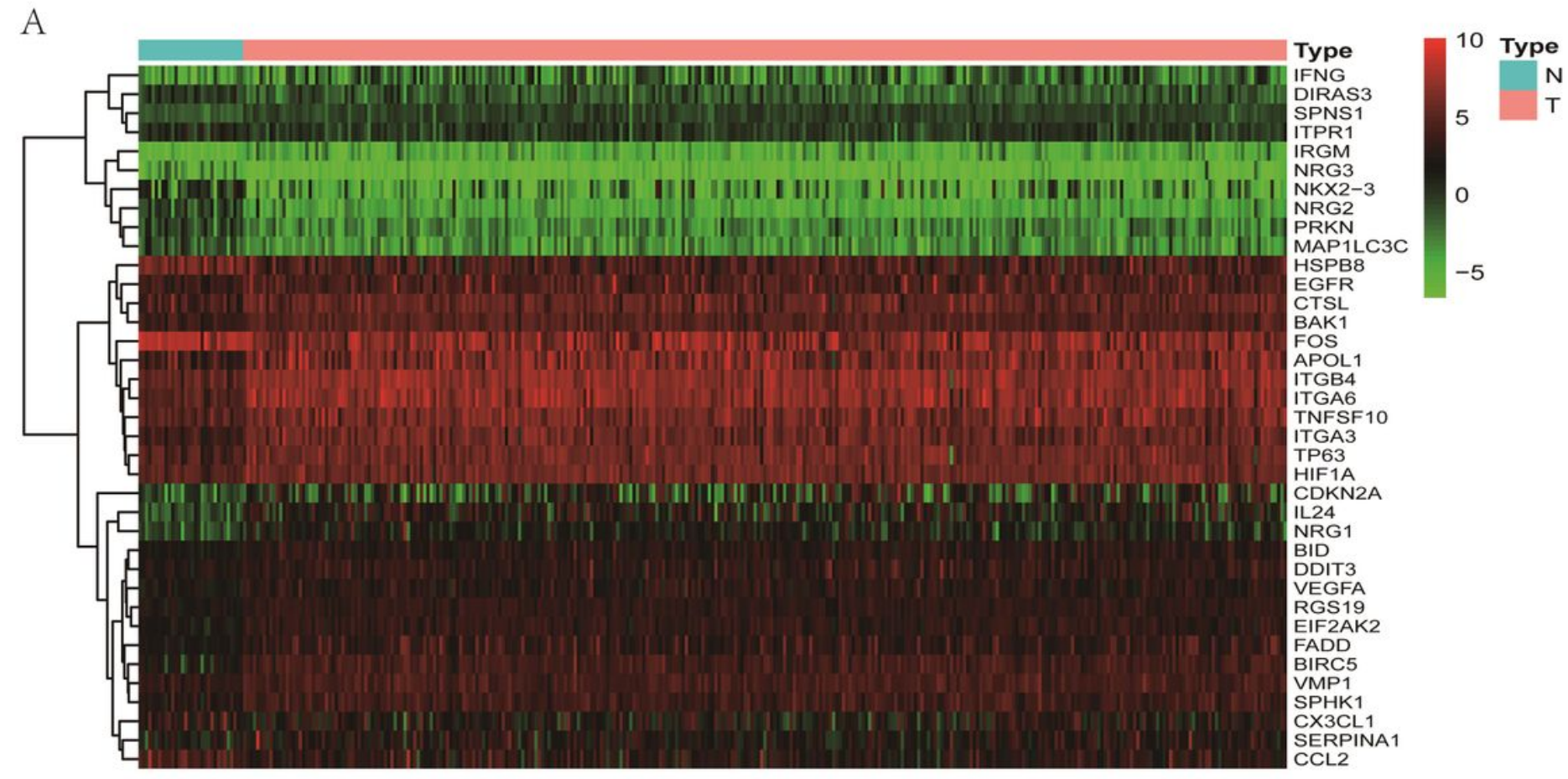

B

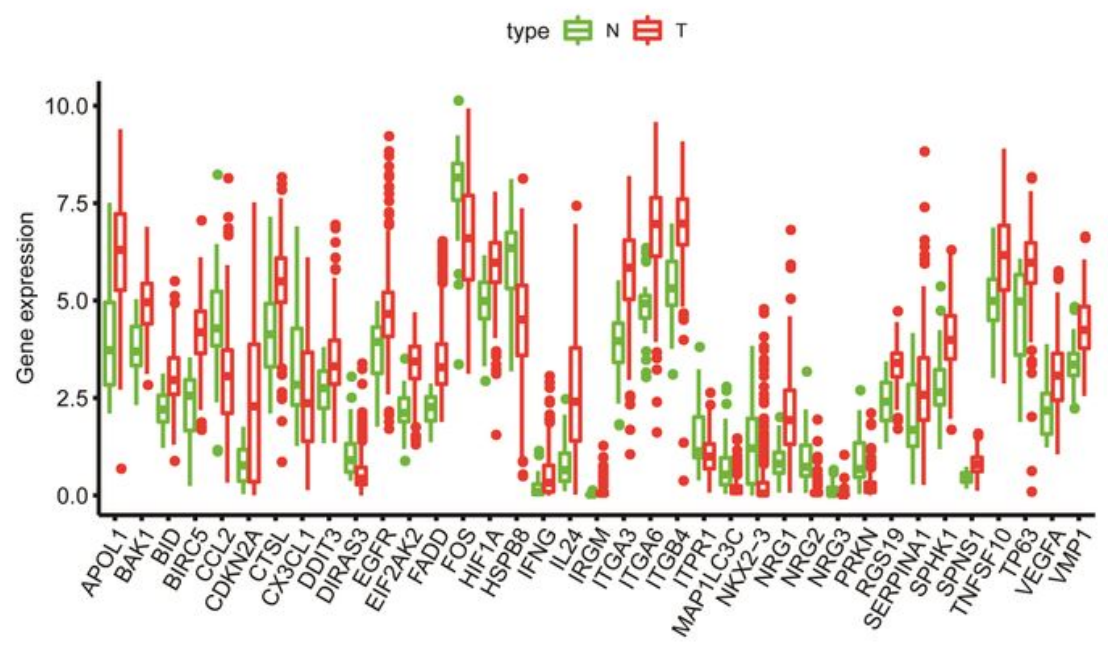

C

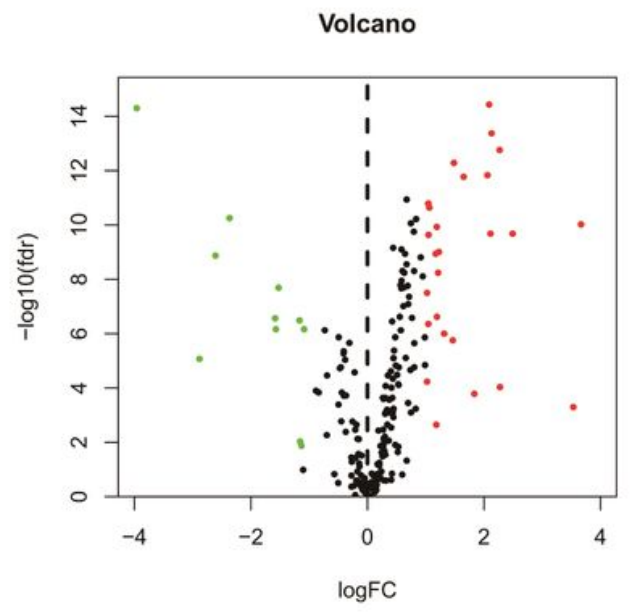

\section{Figure 1}

Distributions of ATGs OSCC. A;The heatmap of 32 normal controls and 316 OSCC samples autophagy related genes expression level. B;Differentially expressed 37 autophagy related genes. C;The volcano of 37 differentially expressed ATGs. 
A

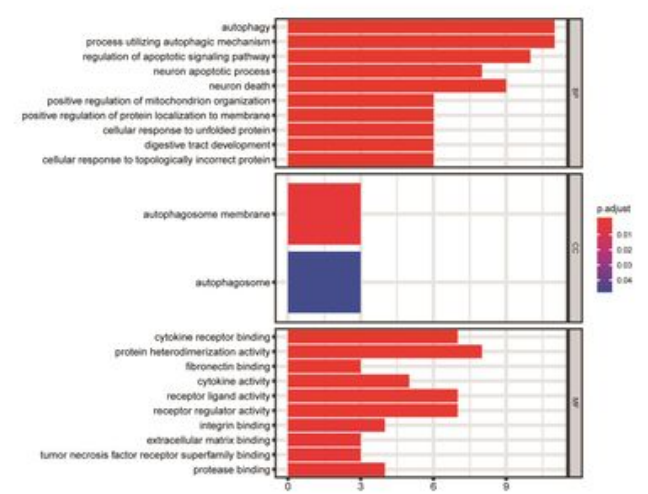

C
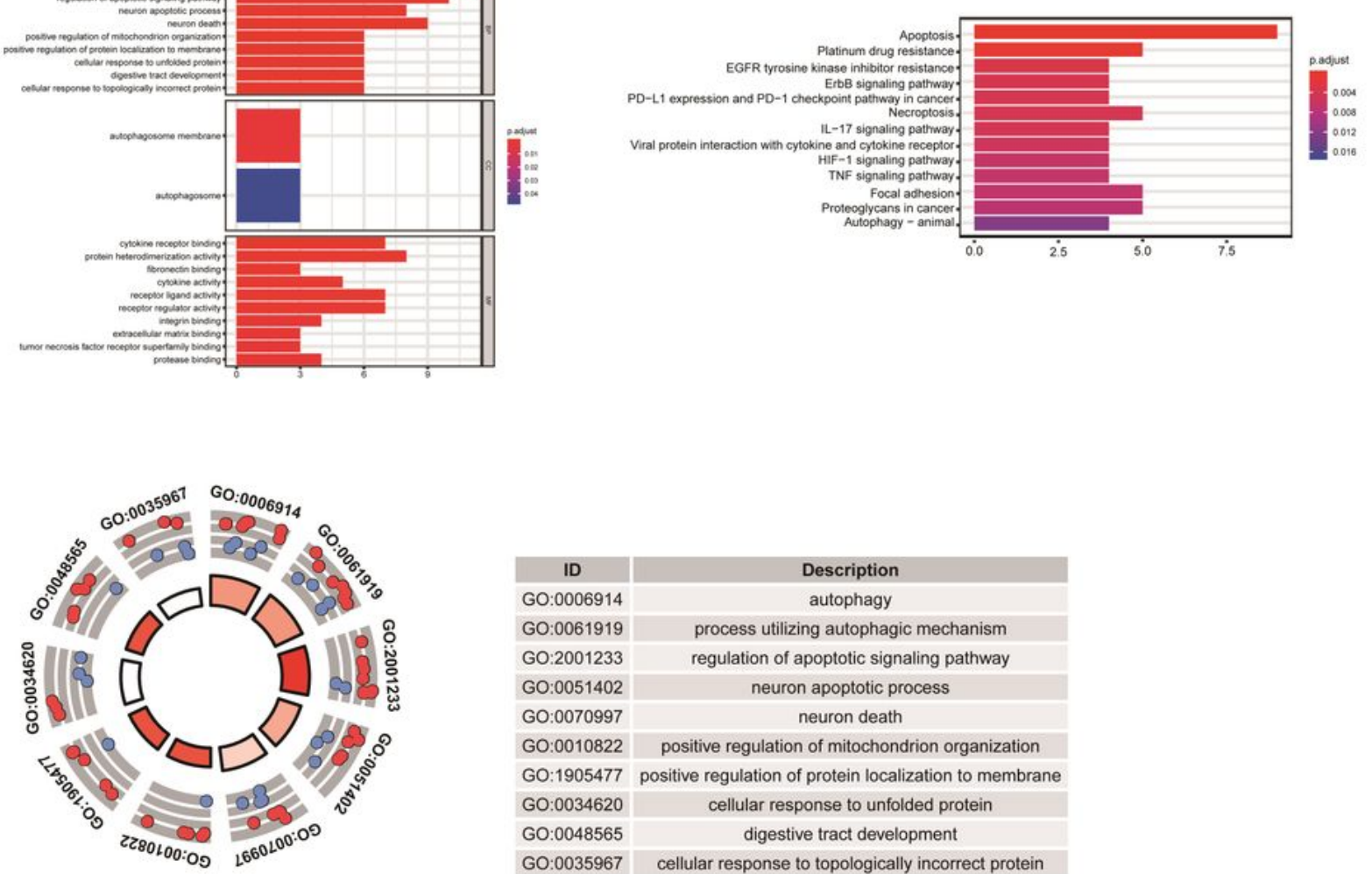

\begin{tabular}{|c|c|}
\hline ID & Description \\
\hline GO:0006914 & autophagy \\
\hline GO:0061919 & process utilizing autophagic mechanism \\
\hline GO:2001233 & regulation of apoptotic signaling pathway \\
\hline GO:0051402 & neuron apoptotic process \\
\hline GO:0070997 & neuron death \\
\hline GO:0010822 & positive regulation of mitochondrion organization \\
\hline GO:1905477 & positive regulation of protein localization to membrane \\
\hline GO:0034620 & cellular response to unfolded protein \\
\hline GO:0048565 & digestive tract development \\
\hline GO:0035967 & cellular response to topologically incorrect protein \\
\hline
\end{tabular}

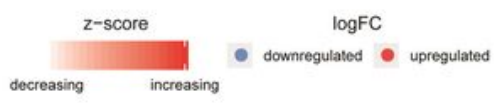

D

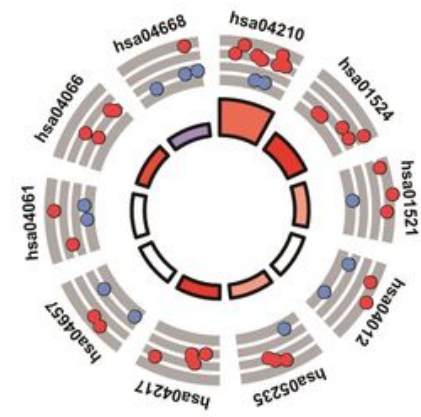

\begin{tabular}{|c|c|}
\hline ID & Description \\
\hline hsa04210 & Apoptosis \\
\hline hsa01524 & $\begin{array}{c}\text { Platinum drug resistance } \\
\text { hsa01521 }\end{array}$ \\
\hline hsa04012 & EGFR tyrosine kinase inhibitor resistance \\
\hline hsa05235 & PD-L1 expression and PD-1 checkpoint pathway in cancer \\
\hline hsa04217 & Necroptosis \\
\hline hsa04657 & IL-17 signaling pathway \\
\hline hsa04061 & Viral protein interaction with cytokine and cytokine receptor \\
\hline hsa04066 & HIF-1 signaling pathway \\
\hline hsa04668 & TNF signaling pathway \\
\hline
\end{tabular}

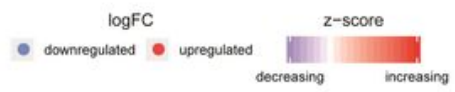

\section{Figure 2}

A; The number of abundant ATGs in each GO category. The $Y$ axis represents the GO categories, while the $X$ axis represents the enrichment score. Furthermore, the color represent $p$-value. B;The most important pathways in differentially expressed autophagy. The $\mathrm{Y}$-axis represents the pathway, the X-axis represents the number of abundant genes, and the color represents the adjusted p-value. C,D;The top 10 related GO function and KEGG pathways were listed. 
A

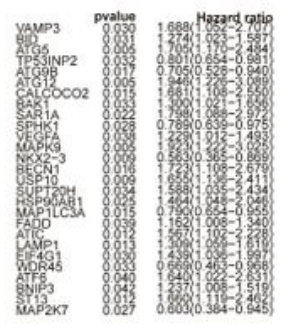

B

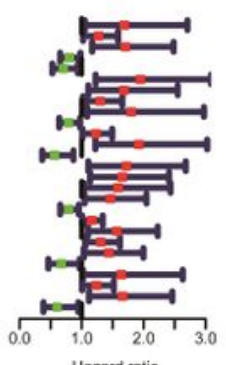

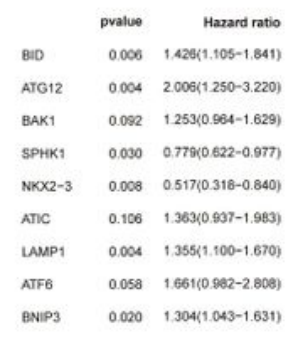

C
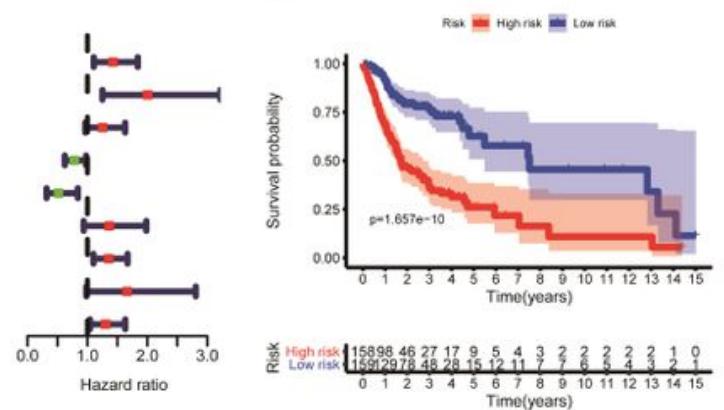

D

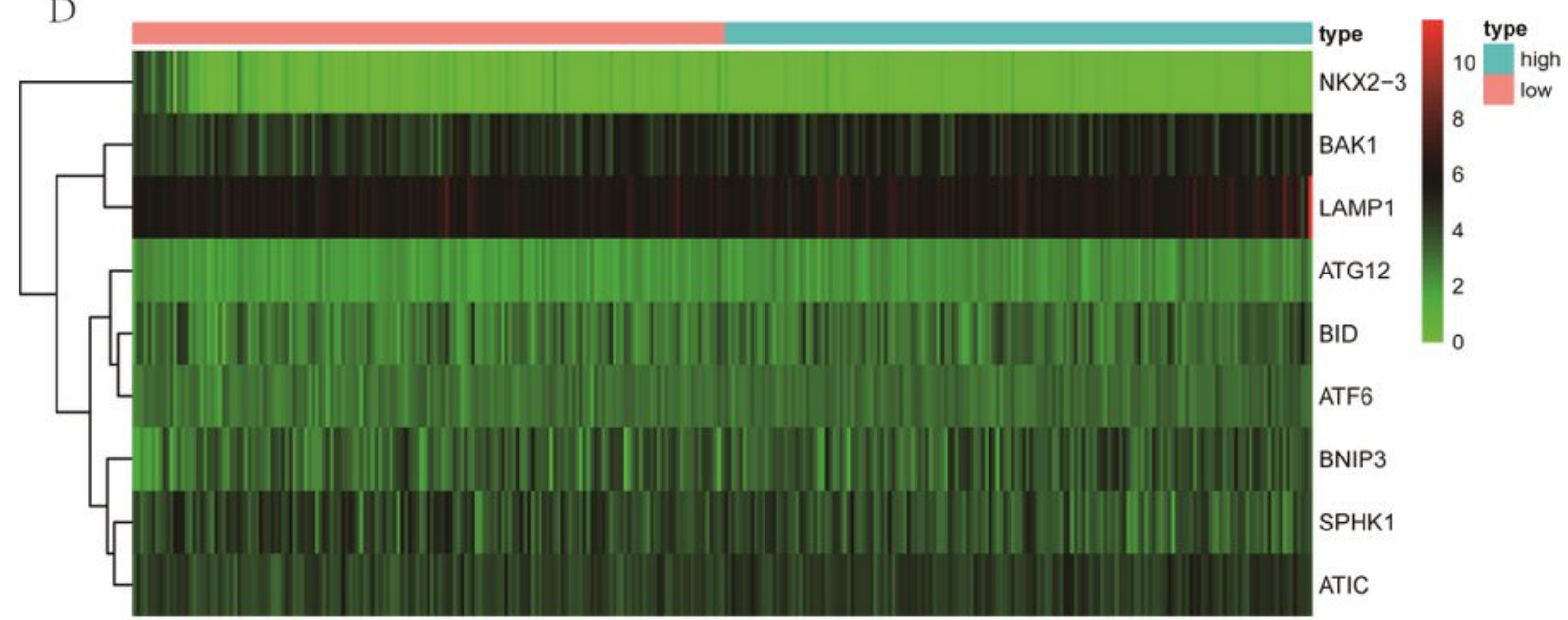

E

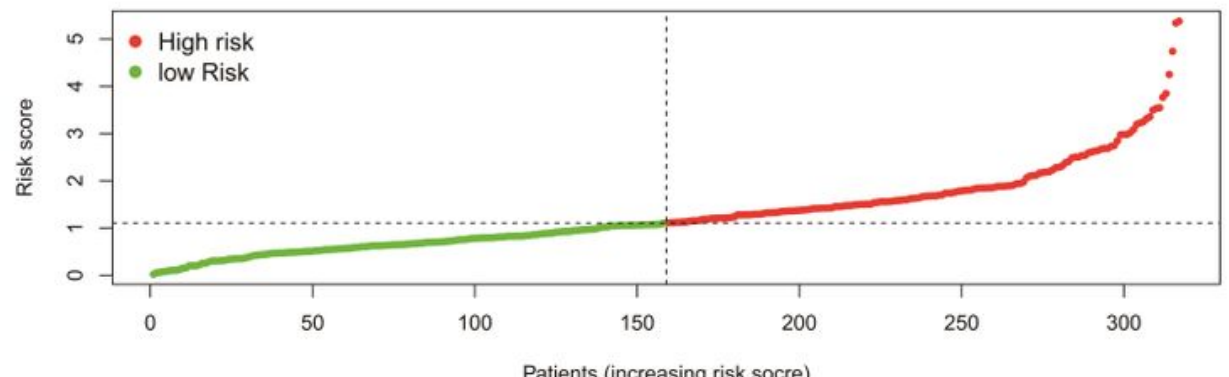

F

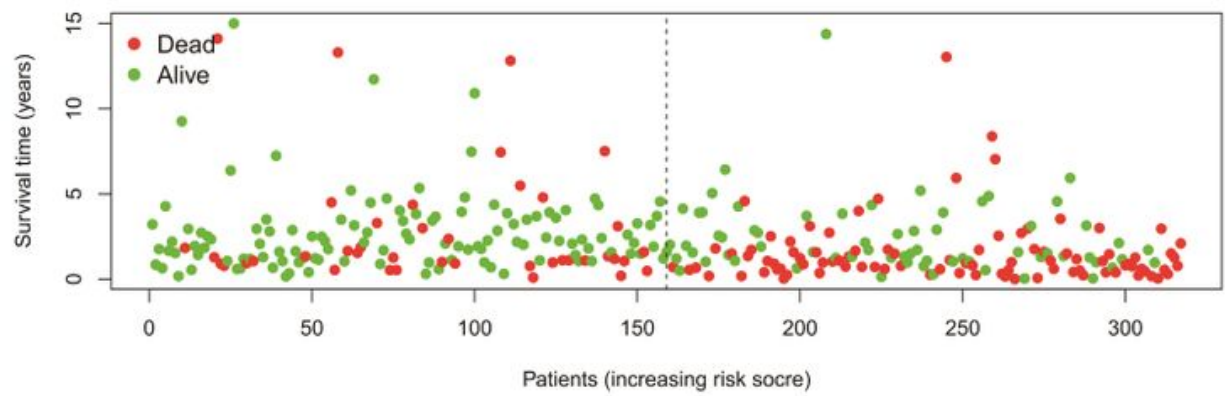

\section{Figure 3}

A;Univariate cox regression based on differentially expressed autophagy genes. B;Cox regression model was constructed on basis of multivariate cox regression. C;Survival analysis was performed according to cox regression formula. D;The heatmap of 9 prognostic factors expression profiles.E,F;Risk score combined with survival time was viaulized in $\mathrm{R}$ softare. 


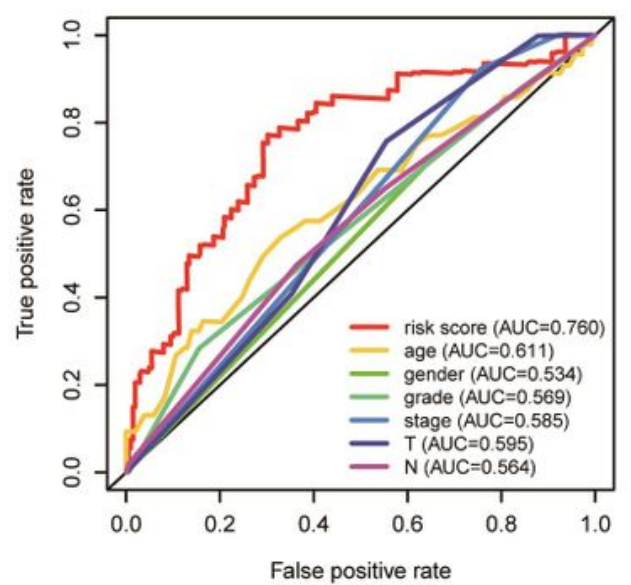

C

$\begin{array}{lrr} & \text { pvalue } & \text { Hazard ratio } \\ \text { age } & 0.005 & 1.025(1.007-1.043) \\ \text { gender } & 0.811 & 0.950(0.626-1.442) \\ \text { grade } & 0.039 & 1.399(1.017-1.924) \\ \text { stage } & <0.001 & 1.786(1.357-2.349) \\ \mathrm{T} & <0.001 & 1.517(1.230-1.872) \\ \mathrm{N} & <0.001 & 1.520(1.219-1.895) \\ \text { riskScore } & <0.001 & 1.995(1.672-2.379)\end{array}$

$\mathrm{D}$

$\begin{array}{lrr} & \text { pvalue } & \text { Hazard ratio } \\ \text { age } & 0.006 & 1.029(1.008-1.050) \\ \text { gender } & 0.364 & 1.236(0.782-1.955) \\ \text { grade } & 0.062 & 1.387(0.984-1.956) \\ \text { stage } & 0.429 & 1.210(0.755-1.942) \\ \mathrm{T} & 0.066 & 1.345(0.980-1.846) \\ \mathrm{N} & 0.254 & 1.172(0.892-1.541) \\ \text { riskScore } & <0.001 & 1.917(1.585-2.319)\end{array}$

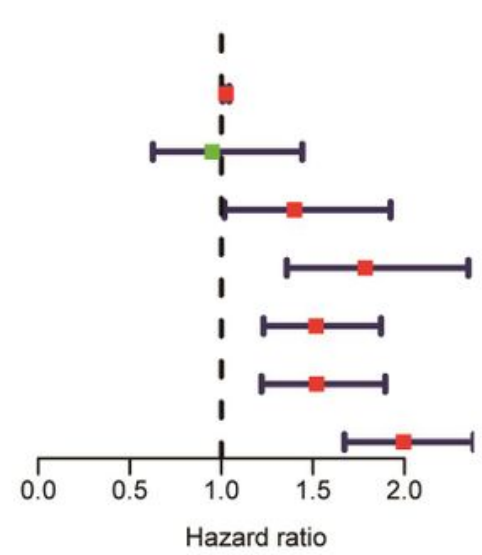

E

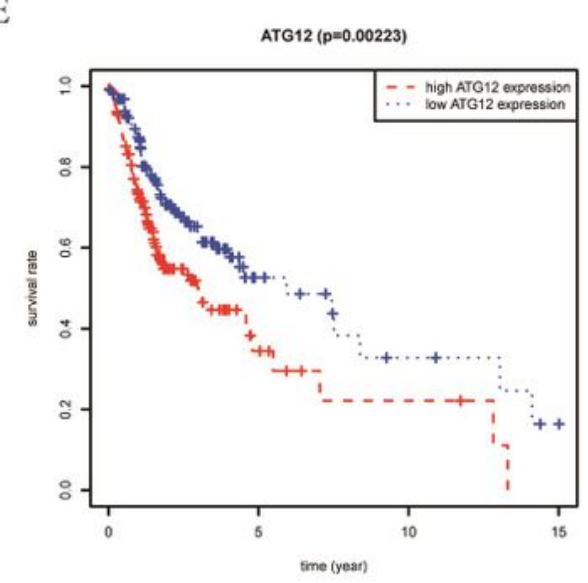

F

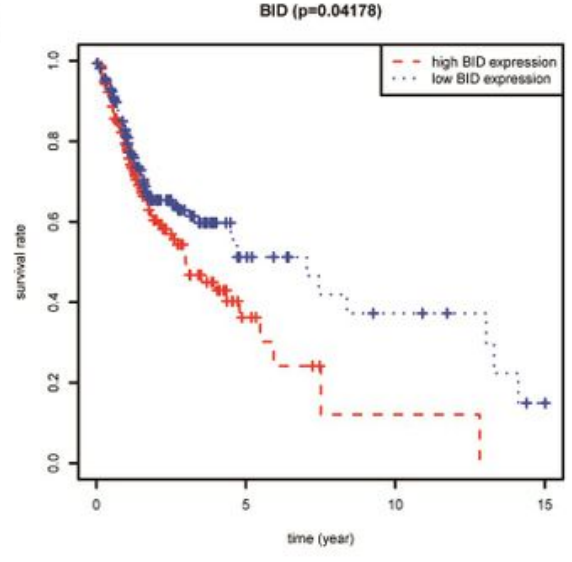

\section{Figure 4}

A;The ROC was plotted according to risk score,and its area Under the Curve (AUC) is 0.76.B;Differences in risk scores between early stage(stage1+2) and terminal stage(Stage3+4).C,D; Univariate and multivariate cox regression analysis reveal whether the risk score can be regarded as indenpendent prognostic biomarker. E,F; Survival analysis according to autophagy related genes enrolled in cox regression model. 

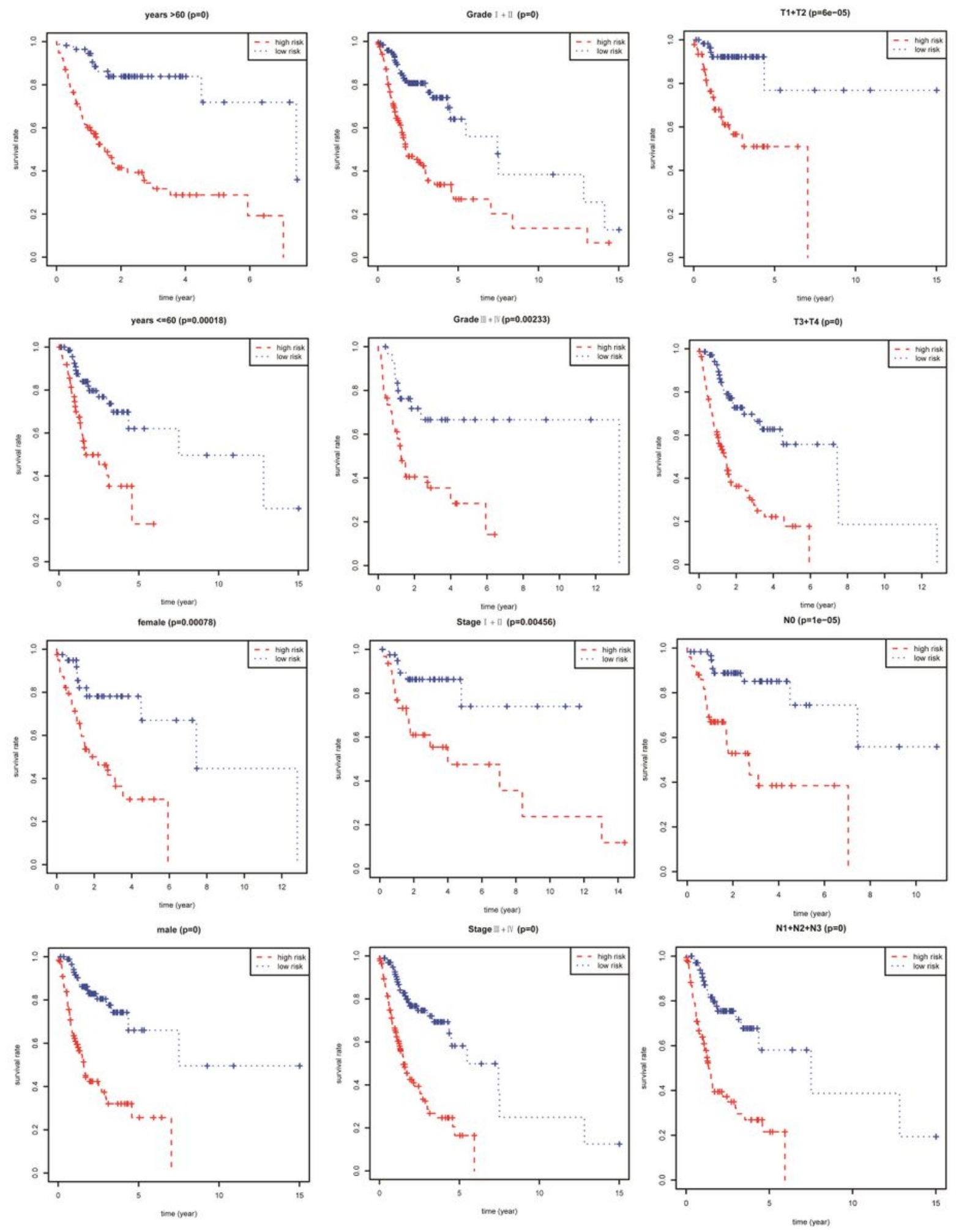

Figure 5

Stratification analysis analysis combined with survival analysis to identifiy the accuracy of the risk score model. 
A
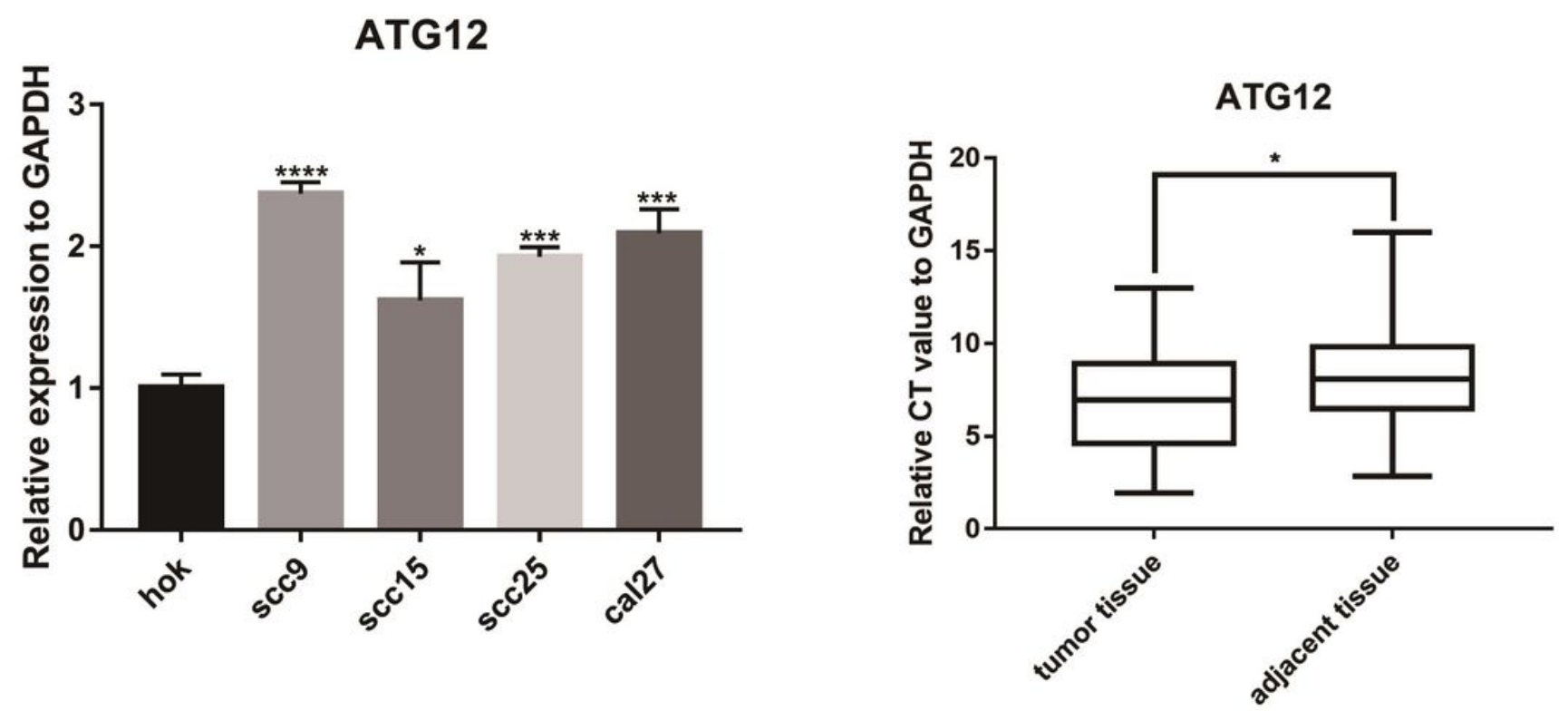

B
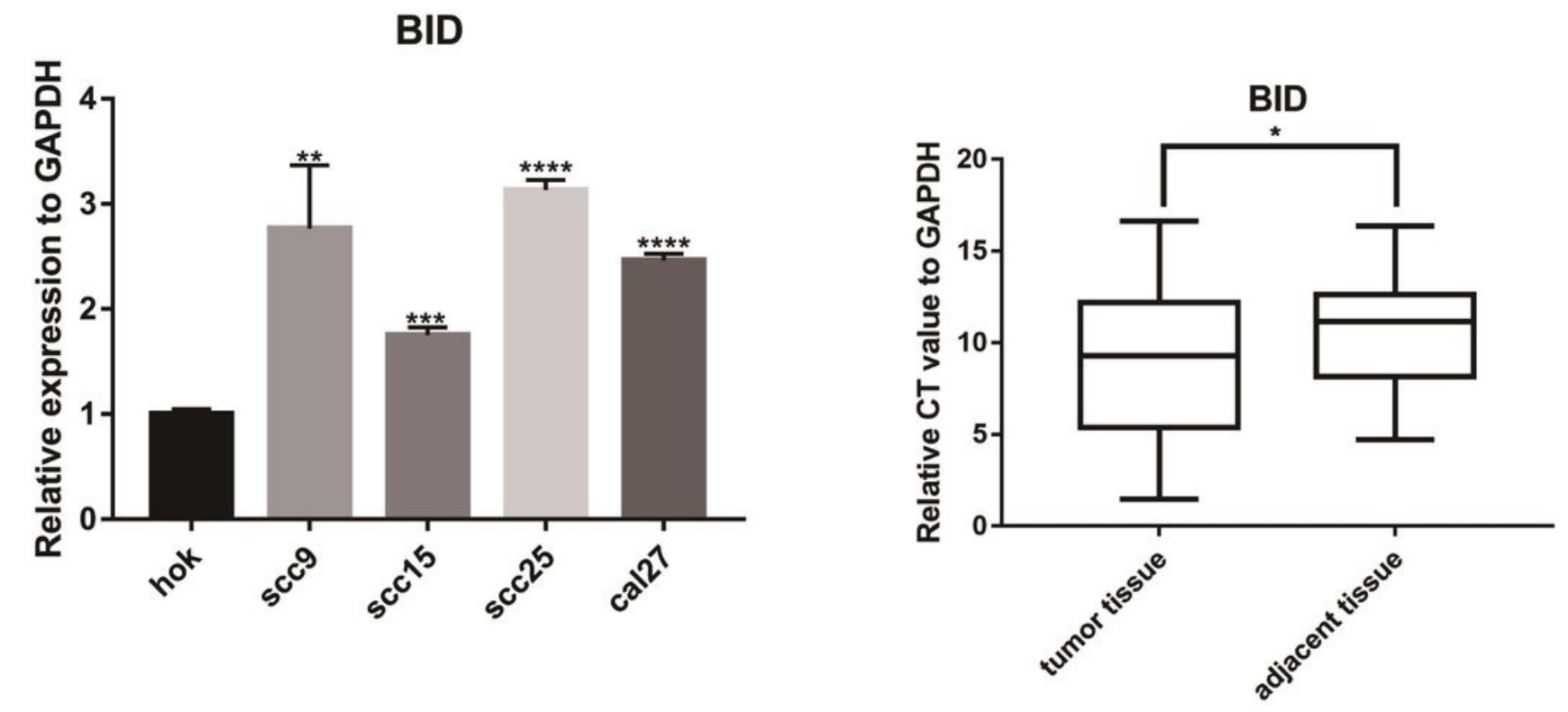

Figure 6

A;ATG12 expression level in OSCC cell lines and tissues. B; BID expression level in OSCC cell lines and tissues. 